\title{
Sharing the viewing experience through Second Screens
}

\author{
Mark Lochrie \\ School of Computing and Communications \\ Infolab21, Lancaster University \\ Lancaster, LA1 4WA, UK \\ +44 (0) 1524510537 \\ m.lochrie@lancaster.ac.uk
}

\author{
Paul Coulton \\ School of Computing and Communications \\ Infolab21, Lancaster University \\ Lancaster, LA1 4WA, UK \\ +44 (0) 1524510393 \\ p.coulton@lancaster.ac.uk
}

\begin{abstract}
Despite the ever expanding forms of digital entertainment and the emergence of consumer recording facilities, allowing viewers to time shift their TV viewing habits, there are still certain TV shows and events that create an audience desire to be part of a mass shared experience. In the past direct inter-audience interaction of such events has been restricted to either a shared location at the time of broadcast or later discussions amongst friends and colleagues often described as 'water cooler moments'. With the advent of online social networks that facilitate status updates these moments can be instantly shared in real-time using mobile phone creating a second screen for interaction with TV. In this paper we investigate the emerging role of mobile phones as the facilitator of second screen for TV. This is achieved through the analysis of tweets for a highly popular UK TV show the X Factor and comparing tweets from this show with other shows from a different format. The results highlight the rich source of information that can be extracted in real-time and how audiences are creating their own parallel narrative of the show through Twitter. This interaction has enormous potential for broadcasters and producers both in terms of reinvigorating live TV viewing and creating new forms of audience interaction.
\end{abstract}

\section{Categories and Subject Descriptors}

H.5.1 Multimedia Information Systems

\section{Keywords}

Mobile, second screen, interactive, television, Twitter, shared experience, narrative, performance

\section{INTRODUCTION}

In the last decade we have seen the introduction and rise of Digital Video Recorders (DVRs) changing the way viewers time-shift and consume their TV entertainment [3]. The initial increase in DVR ownership resulted in a decrease in watching live TV as there wasn't a real-time need to consume at the time of broadcast. As in the past if you wanted to discuss what you have just watched, you had to wait till the next day at work, typically around the refreshments, thus the term "water cooler moments". The

Permission to make digital or hard copies of all or part of this work for personal or classroom use is granted without fee provided that copies are not made or distributed for profit or commercial advantage and that copies bear this notice and the full citation on the first page. To copy otherwise, or republish, to post on servers or to redistribute to lists, requires prior specific permission and/or a fee.

EuroITV'12, July 4-6, 2012, Berlin, Germany.

Copyright 2012 ACM 978-1-4503-1107-6/12/07...\$10.00. increase and popularity of Saturday night prime time competition and reality shows depend on facilitating some form of viewer engagement [3] bringing about the requirement a return channel between program and viewer. In terms of the device to utilise for such a purpose the obvious choice is the ubiquitous mobile phone, as an increasing amount of these devices have low barriers to entry in accessing data services. This access is facilitated by cheaper data plans on mobile networks and widespread connectivity using home Wi-Fi networks enabling new methods to engage users through web or rich application based content. These so-called 'second screen devices' are now becoming the first choice for multitasking whilst watching TV. Yahoo and Razorfish surveys report that $88 \%$ were mobile multitasking whilst watching TV $(15 \%$ of which were on their mobile devices the entire duration of the show and $38 \%$ using their mobile device for consuming content relating to the show).

At the same time we have seen the dramatic rise of social media services, such as Facebook, and Twitter that link into existing social networks, being used to curate forums for debate around a range of topics including TV shows. Twitter in particular with its ability to share topics through 'hashtags' and 're-tweets' are now being used by audiences to discuss TV programmes in almost real-time. Twitter recently introduced new ways to discover and engage with current social buzz content on the mobile device through its "Twitter Discovery" service. Twitter has also made it even easier to re-tweet, mention and to include hashtags with prepopulated tweet windows. This further bridges the gap between the viewer and the TV show, by providing a means of users to browse stories and read tweets around the story.

It is important here to differentiate the use of Twitter by TV programs to enable audiences to participate within the program (effectively making it an alternative to text messages) and the general discussion amongst the audience within the Twitter stream itself which will be the main thrust of the research in this paper.. Broadcasters increasingly embracing Twitter as a means of extending their audience relationship and to drive viewership by integrating: on screen hashtags as closed captions, TV personalities live tweeting during aired show, vote by tweet (DM), tweets used as part of the TV show, specific show related standalone applications and follow me as closed captions (usually depicted as flashy banners that are located either along the footer or in the corners of the screen).

Studies from Twitter $^{3}$ suggests that when broadcasters combine the real-time elements of Twitter, there is a direct and immediate increase of viewer engagement from anywhere from two to ten times more the amount of mentions, follows and hashtags used whilst the show airs. This is highlighted by the 2010 Grammy Awards, which saw a 35\% increase on viewing figures from 2009, one of the suggested reasons for this increase is the integration of social media in the 2010 event. Confirmed by the Mobile World 
Congress 2011 in Barcelona, during Dick Costello's keynote which outlined the future of Twitter in the TV entertainment business, adding value to live TV shows and deterring viewers from DVRs, as 'real-time' viewing is preferred so they could tweet.

\section{RELATED WORK}

Whilst previous research has focused on how such devices can best facilitate the provision of shared content, enabling recommendations from friends, or sharing an interactive link with friends, few have addressed real-time interaction between the whole viewing audience. Direct inter-audience interaction amongst large groups could provide a greater sense of shared experience for the audience, as it allows for the free and frank interactions such as those seen on fan forums and discussion boards $[3,4,5]$.

Although the use of Twitter within the American Presidential Debates was studied [7], it was not performed in real time on individual tweets but rather through later the time synchronised rank analysis of terms used both tweets and transcript of what each speaker had to say. We have also seen similar studies analysing the effects that in vision closed captioning has on the Twitter stream i.e. how people were described [6] on TV and what impact this had on ranked terms that appeared in the Twitter stream [6, 7]. While both these studies are based on spoken dialogue the study presented in this paper considers TV shows that present a more visual spectacle and examines whether this changes the influence of the discussion within Twitter.

Therefore to gain a better understanding of this activity we developed a system to record and analyse in real-time all the tweets associated with the highly popular UK TV show 'The $\mathrm{X}$ Factor' during the 2010 series.. It was subsequently used to collect tweets on the 2011 shows of The X Factor and The X Factor USA. The $\mathrm{X}$ Factor USA which was the first show to directly integrate Twitter into its show, by utilising Twitter's Direct Messaging (DM) service for registering contestant votes and incorporating hashtags on screen for the show, the contestants and judges and to promote live tweets from the judges.

\section{BACKGROUND TO STUDY}

This paper provides a detailed analysis of The X Factor in particular the 2010 and 2011 series and its recently aired American counterpart. The X Factor is a reality TV show in which aspiring pop singers compete against each other for a recording contract. The shows format differs from other singing contests by characterising each contestant into groups mentored by one of the judges; each judge is assigned a category and then aids the contestant with song selection and styling. The contestants are competing for the public vote in the form of phone calls and text messages. Generally the judges make the final decision between the bottom two contestants after the public vote. In its opening weekend X Factor attracted 11.1 million viewers representing a $48 \%$ share of the national TV viewing audience and rose to 19.1 million viewers for the final, which is $61.9 \%$ of the national audience, making it the highest rated television episode of 2010 in the UK.

To enable us to perform this study Twitter's Streaming API was used to capture all tweets that contain the \#xfactor hashtag in its raw form. The tweet data is then parsed into different tables (tweet data, mentions, tags, urls and users), this allows deeper analysis of the tweet content, its source, the tags used, any urls included and who tweeted it. To analyse the use of mobile for a 'second screen' it was also important determine the origin of each tweet. Based on the tweet data collected volume information can thus be calculated on a minute-by-minute basis.

\section{TWEET DATA RESULTS \\ 4.1 The $X$ Factor 2010 analysis}

Due to the sheer volume of data generated during this research project we limit data presented in this paper to the build-up and coverage of the finals weeks and live show. In terms of structure we see a definite narrative in relation to volume of tweets related to the contestants that correspond directly to the running order of the show.

Having evaluated the narrative of the show through Twitter it is interesting to compare this with other measures such as the phone votes received for each contestant during the wholes series (Total votes cast: $15,448,019)^{1}$. What is very clear is that Matt had the most support from beginning of the show and interestingly the final phones for Matt and Rebecca were $44 \%$ and $38 \%$ which is a greater difference than the weekly tweet results although confirms greater support for Matt.

\subsection{The X Factor UK and USA 2011}

The $\mathrm{X}$ Factor franchise finished airing on America's FOX network, running from $21 / 09 / 2011$ to $22 / 12 / 2011$, that averaged around 12 million viewers per episode. It was also the eighth year The X Factor appeared on British television, although the show didn't manage to retain the average UK viewing figures from its previous year (14.13 million) it still achieved an average of 12.03 million (higher than its American counterpart).

Similar to the UK series, the viewer engagement surged once the live shows started and they introduced vote by tweet, the vote by tweet are kept private as they are sent via DM. A similar trend was seen in The X Factor UK 2010 where the youngest contestants were the most popular amongst social network discussions. The US shows young contestant Astro, Rachel Crow and Melanie Amaro attracted the highest peaks of discussion on social networks. During the first week of vote-by-tweet the youngest contestant Astro (15 years old, an urban rap singer) was the most popular amongst Twitter users (this could be down to the demographics that use social network and watch these types of TV shows thus having more in common and can relate more with the song choices by the contestant).

\subsection{Tweeting Platforms}

\subsubsection{The X Factor UK 2010 tweet data}

In order to analyse the platforms used to create tweets source, a classification system of MOBILE, NON-MOBILE and MIXED was adopted from previous research [3] as limitations of Twitters metadata which details the agent used rather than the exact platform [3].

Figures 1.A (final week snapshot 05/12/2010 - 13/12/2010) illustrates the role of mobile for tweeting during the actual live TV shows and there is clearly a big difference between the performance show (Figure 1.B 11/12/2010 19:00 - 21:00) and the results show (Figure 1.C 12/12/2010 19:30 - 21:30). It seems likely this is due to the difference in show formats as one is performance led and the other a time slot filled with pieces to

\footnotetext{
${ }^{1}$ Twitter Blog http://blog.twitter.com/2011/05/watching-together-twitter-and-tv.html
} 
make a show before the phone in results are announced. This could suggests that there is perhaps less combined viewing on the main TV screen and thus an increased need for the second screen although this would need to be confirmed with more targeted research. Another interesting distinction is that viewers are more likely to watch the performance show and tweet during the commercials, whereas on the results show, viewers were more likely to engage with discussion and express their support on who they want to win during the show.

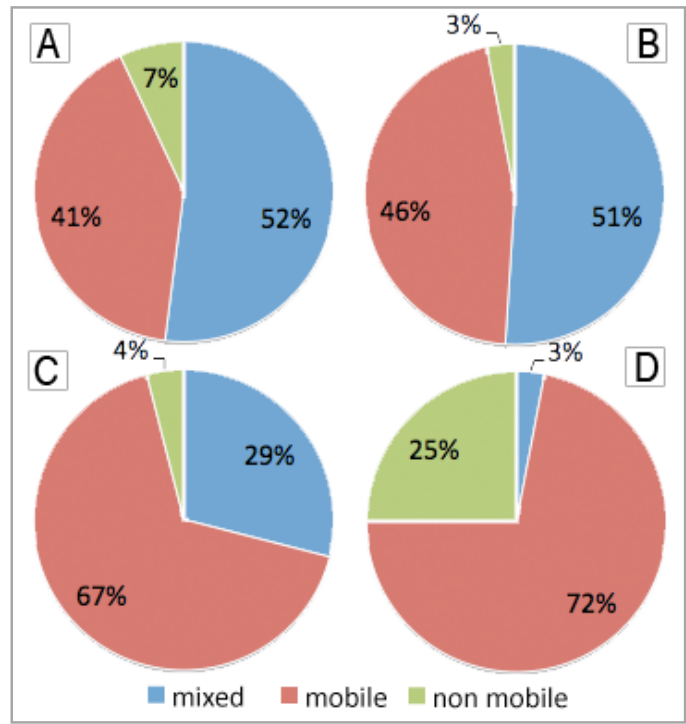

Figure 1. Tweet device data for mobile, non-mobile and mixed platforms

\subsubsection{The X Factor UK 2011 tweet data}

As previously mentioned, we captured and analysed a range of TV show formats, ones similar to The $\mathrm{X}$ Factor and some completely different in terms of show format. It was interesting to re-analyse the following year's (2011) mobile impact on the same show, as this time around social media has more of the spotlight, integrated more effectively into the shows programme. Unlike the US counterpart, the UK show hadn't moved over to vote-bytweet, but by researching the impact it had in the US show, it would make a reasonable presumption similar features will appear in the UK show in the not too distant future. However the UK show definitely made more attempts at integrating the show on Twitter; by having tweet names and hashtags appear on screen as closed captions, a rise in judges and contestants joining and using Twitter, connecting with their audience not just through the TV screen impacting on audience voting influences.

Similarities were apparent when comparing the shows (both seasons of The X Factor 2010/2011). A snapshot of the final show was taken to identify which tweeting platforms were commonly used. Figure 1.D represents the percentages of platforms used to tweet during the live final results show (11/12/2011 19:00 22:00). The biggest difference is mixed platforms have decreased, this could be related to the fact Twitter has improved its tweet sourcing data. Also notably is the increase in mobile tweeting platforms. This increase can be associated with how the producers closely integrated Twitter more into its show with on screen captions, contestants and judges on Twitter themselves, the integration of Twitter into the OS (Operating System) in iOS devices and the increase could also be related to the growth in tablet sales last year ${ }^{2}$.

\section{SECOND SCREEN FOR TV: FUTURE}

If we look more closely at the demographic for mobile Internet users that use their smartphone or tablet whilst watching TV (86\%) $92 \%$ are $13-24$ year olds. Since conducting this research a number of mobile applications have appeared attempting to bridge the gap between TV show and its audiences. For example, $X$ Factor reintroduced their 'Tap to Clap' feature in the shows official application.

Pepsi recently sponsored The X Factor USA and developed "The Pepsi Sound Off" [//pepsisoundoff.com] adding a gamification element to boost viewer discussions. The platform allowed fans to chat about the show, contestants and judges, creating a narrative of the show amongst viewers, each viewer would earn points for every discussion they make and players are then ranked on leaderboards to compete with their friends.

Arguably the best example so far is 'Heineken Star Player' [//heineken.com/gb/ucl/star-player/] which encourages viewers to anticipate an outcome of in-game events e.g. where the ball will end up on a penalty. The viewers are awarded points if they successfully predict the outcome and are represented in leaderboards and badge system to reward their participation.

Recent announcements that Mark Burnett (producer of The Apprentice) and ACTV8 [//actv8.me] are developing ways of reinvigorating and innovating viewer engagement using the traditional smart phone and tablet. Mobile platform users will have the platform to interact, socialize, consume, share and participate in real time, all while watching their favourite TV shows. The application synchronizes a user's mobile device to any live or recorded video broadcast providing rich interactive content delivered straight to the users' mobile device relating to the programme they are watching. Not only does the application synchronised with the TV programme it also links into users' social networks, thus providing a platform for users to voice their opinions. ACTV8 have also realised the potential for broadcasters and advertisers by bringing the viewer directly into the TV programme providing programme enhancements, line extensions, quizzes, trivia, gaming and products seen in TV shows and also during commercials. Not only will this type of viewer engagement be rewarding for broadcaster and advertiser the application attempts to reward its users for their participation with content, including special offers and coupons from many of the leading TV advertisers.

Similarities can be seen with zeebox [//zeebox.com/] a combination of social networking, live programme information, TV remote control and real-time viewing figures application. The mobile and desktop application allows viewers to socialise immerse and interact whist watching TV. Both applications outlined show great potential for leading the way for utilising mobile devices as second screens.

\section{CONCLUSION}

In this research we presented a study into how the social networking service Twitter is increasingly being used as a channel for real-time inter-audience interaction for TV and implications for the role of mobile as the 'second screen'. To facilitate this

\footnotetext{
2 The Guardian - UK tablet sales http://www.guardian.co.uk/technology/2011/dec/25/ipad-tabletdominates-third-quarter-2011
} 
research a system was created that allowed the storage and analysis in real-time of tweets on a minute-by-minute basis for the highly popular UK TV show the X Factor.

The data collected illustrates the high levels of interaction, already developing in the use of Twitter as an inter-audience forum, around TV and how the narrative of the show is played out through this interaction. Whilst the narrative is obviously driven in time by events in the show, many of the tweets also exhibit a great deal of humour, and irony which could be considered almost performative in nature, possibly demonstrating the notion of performing to the 'invisible audience' [1]. Compared with the previous study on the Presidential Debate which showed little correlation between what was in the tweets and what was said by the debaters in this format there was often a significant correlation between what was appearing on screen and the tweets suggesting a confirmation of the study involving closed captions that visual imagery has a more significant impact for this dual mode interaction.

In terms of using Twitter as a possible guide to predicting events it is clear that whist in this case the Twitter stream did replicate the phone voting in the majority of cases, anomalies do still occur suggesting that there are significant differences, in the demographic of the online community compared with those actually voting. Therefore producers and broadcaster wishing to use real-time Twitter analysis for audience interaction, should consider how best to weight the importance of such interaction in relation to their whole audience, as we have found out that different levels of audience interaction depends on the type of show in question.

As stated previously the tweet data captured provides an indication of who was being talked about and when, however it does not provide us a platform to analyse if this is positive or negative. To perform a deeper analysis the tweet language would have to be taken into consideration, this would be very problematic due to Twitter's status update constraints (140 characters) as in order to perform a deeper language analysis more words are required [8]. Twitter's API does provide a simple approach to this by using Emoticons (happy/sad faces depicted by punctuations i.e. :) (:), however this method for detecting emotion in tweets is limited, as the majority of tweets captured in this study do not contain Emoticons to express emotion.

As this research did not consider the motivations of the individual involved it would seem appropriate that further research on this topic addressed these motivations more specifically.

Whilst this study included tweets from a variety of mediums the information obtained about the clients used to up-load the tweets indicates over $40 \%$ are from mobile, which is consistent with the figures reported by Twitter. Further, as reported widely in the media in February 2011 an IDC report showed that smartphone manufacturers shipped 100.9 million devices in the fourth quarter of 2010, while PC manufacturers shipped 92.1 million units worldwide in other words smartphones outsold PCs for the first time ever. As this trend is likely to continue then accessing internet services through mobile are likely to increase dramatically in the near future [2].
Overall this study highlights that mobile phones are already becoming the second screen for TV but not through broadcaster provision of personalised services, or service providers enabling them to act as a new form of remote, but rather by audiences themselves creating their own forums for inter-audience interaction. It is therefore important for broadcaster and producers to be able to better understand the nature of this interaction and how it may be utilised to create new forms of interactive TV and thus is a topic requiring further research.

\section{ACKNOWLEDGMENTS}

Many thanks to Lancaster University Network Services (LUNS) for providing a high bandwidth connection used to stream tweets from Twitter.

\section{REFERENCES}

1. Boyd, D., Why Youth (Heart) Social Network Sites: The Role of Networked Publics in Teenage Social Life, MacArthur Foundation Series on Digital Learning - Youth, Identity, and Digital Media Volume (ed. David Buckingham). Cambridge, MA: MIT Press, 2007.

2. Eronen, L. \& Vuorimaa, P., User Interfaces for Digital Television: a Navigator Case Study. Proceedings of the working conference on advanced visual interfaces - AVI 2000. Palermo, Italy, 23-26 May 2000, pp.276-279. New York: ACM Press.

3. Lochrie, M. \& Coulton, P., 'Tweeting with the telly on!: Mobile Phones as Second Screen for TV', Paper presented at Social Networks and Television: Toward Connected and Social Experiences at IEEE Consumer Communications \& Networking Conference, Las Vegas, United States, 14/01/12 17/01/12,

4. Park, J., Blythe, M., Monk, A., and Grayson, D. 2006. Sharable digital TV: relating ethnography to design through unuseless product suggestions. In extended abstracts on Human factors in computing systems, 119-1204. 2006.

5. Robertson, S. Wharton, C. Ashworth, C. and Franzke, M., 1996. Dual device user interface design: PDAs and interactive television. In Proceedings of the SIGCHI conference on Human factors in computing systems: common ground (CHI '96), Michael J. Tauber (Ed.). ACM, New York, NY, USA, 79-86.

6. Sayaka Akioka, Norikazu Kato, Yoichi Muraoka, and Hayato Yamana. 2010. Cross-media impact on twitter in japan. In Proceedings of the $2^{\text {nd }}$ international workshop on Search and mining user-generated contents (SMUC '10). ACM, New York, NY, USA, 111-118.

7. Shamma, D.A. Kennedy, L. and Churchill, E.F., Tweet the debates: understanding community annotation of uncollected sources. In Proceedings of the first SIGMM workshop on Social media (WSM '09). ACM, New York, NY, USA, 3-10.

8. Simm, W.; Ferrario, M.-A.; Piao, S.; Whittle, J.; Rayson, P.; , "Classification of Short Text Comments by Sentiment and Actionability for VoiceYourView," Social Computing (SocialCom), 2010 IEEE Second International Conference on , vol., no., pp.552-557, 20-22 Aug. 2010. 